\title{
Article
}

\section{Analysis and numeric of mixed approach for frictional contact problem in electro-elasticity}

\author{
M. Bouallala ${ }^{1,2, *}$, EL-H. Essoufi ${ }^{1}$ and A. Zafrar ${ }^{1}$ \\ 1 Univ. Hassan 1, Laboratory MISI, 26000 Settat, Morocco.; e.h.essoufi@gmail.com(E.E); zafrar.abd@gmail.com(A.Z) \\ 2 Cadi Ayyad University, Polydisciplinary Faculty, Department of Mathematics and Computer Science, B.P. 4162 Safi, \\ Morocco.
}

Received: 14 February 2020; Accepted: 28 March 2020; Published: 30 March 2020.

\begin{abstract}
This work handle a mathematical model describing the process of contact between a piezoelectric body and rigid foundation. The behavior of the material is modeled with a electro-elastic constitutive law. The contact is formulated by Signorini conditions and Coulomb friction. A new decoupled mixed variational formulation is stated. Existence and uniqueness of the solution are proved using elements of the saddle point theory and a fixed point technique. To show the efficiency of our approach, we present a decomposition iterative method and its convergence is proved and some numerical tests are presented.
\end{abstract}

Keywords: Piezoelectricity, Coulomb friction, Signorini condition, Variational inequality, Fixed point process, Mixed variational formulation.

MSC: 49J40, 47H10, 65K05, 74M10, 74M15, 35J70, 37C25.

\section{Introduction}

$\mathbf{T}$ he numerical study of the piezoelectric [1,2] contact problems [3-7] presents a great challenges, because the non coercivity and non differentiability of some terms. The linear term coupling the mechanical field and electric potential is non coercive and non symmetric. The term corresponding to the friction is convex and non differentiable, in the variational formulation in one hand. In the other hand, the non-linear coupling mechanical field and frictional contact, it can be shown in the norm of the tangential component of the mechanical field present in the frictional function.

To overcome these difficulties, the authors develop some methods like finite elements method [8,9], penalty method and fixed point method [4]. The most and sufficient method for this type of problem is the ones based on convex duality [10] and the introduction of Lagrange multipliers [11-13]. Primal-dual active sets strategy, which is an equivalent to infinite dimensional semismooth Newton method, is applied in [14] while in [15] the author propose a numerical approximation and based Uzawa block relaxation method. Alternating directions method of multipliers (ADMM) is based in [16].

In mechanic of structures one is always interested in the determination of the stress tensor $\sigma$ more than the mechanical displacement itself. Methods have been developed for calculate an approximation of $\sigma$ from $u$ and the drawback is not easy to build an approximation space of tensors satisfying the equilibrium relations and required regularity. A mixed variational formulation have been developed to handle this difficulty. Concerning the piezoelectric contact problems, mixed formulation were developed in [17-19].

In this paper, we introduce a mixed variational approach based Lagrange multipliers which describes the static frictional contact between a piezoelectric body and non-conductive foundation. The standard mixed variational formulation of contact problems is formally in the following form $[17,18,20]$ :

$$
\begin{aligned}
& a(u, v)+b(v, \lambda)=(f, v), \quad \forall v \in V, \\
& b(u, \delta-\lambda) \leq 0, \quad \forall \delta \in \Lambda,
\end{aligned}
$$

where $a(\cdot, \cdot)$ is symmetric, coercive and the term $b(\cdot, \cdot)$ coupling the normal and tangential Lagrange multipliers. This coupling is difficult to handle numerically and for model describing the contact problem with 
friction it is important to use the decoupling form to identify the slid and slip on the contact zone. Moreover, when the model (1) describe problem with electroelastic body the bi-linear form $a(\cdot, \cdot)$ become not symmetric.

The idea consists in decoupling the contact from the friction by introducing two convex sets, one is reserved to the contact multiplier and the second is stated for the friction. This approach leads to decoupled inequalities in mixed variational problem. Since $a(\cdot, \cdot)$ is not symmetric we follow the standards techniques and steps based on $[17,18]$ but with different fixed point map (Step 3 in the proof) and hence more analysis is needed to get the existence and uniqueness result. The resulting problem is an system by blocks of two unknowns (displacement and potential), regarding its structure and the form of blocks we use Gauss elimination technique and then we obtain a Schur complement in the matrix corresponding to displacement subproblem. It is well known (see $[16,21]$ ) that this technique allows a suitable preconditioner for conjugate gradient method employed to solve the resulting symmetric and positive definite system.

To prove the efficiency of this approach, we state suitable numerical fixed point scheme. The convergence is proved basing abstract perturbed problem and fixed point process, Banach fixed point is hence applied. For details concerning the mathematical tools we refer to [22-25].

The paper is structured as: In Section 2, we present the model of equilibrium process of the elastic piezoelectric body in frictional contact with a non-conductive foundation,we introduce the functional spaces for various quantities, list the assumptions on given data and derive the weak formulation of the problem. In Section, 2.2 we state and prove our main existence and uniqueness result, Theorem 1. The proof of these theorem are carried out in several steps and are based on an abstract result in the study of elliptic variational inequalities and Banach fixed point technique. The successive iterative method is detailed followed by the convergence result in Section 4. In Section 5, we conclude with finite element discretization and we give some numerical experiments by simple example .

\section{Problem setting and main results}

\subsection{Problem setting}

The piezoelectric body occupies in its reference configuration (initial configuration) the domain $\Omega \subset \mathbb{R}^{d}$, $d=2,3$. We suppose that $\Omega$ is bounded with a smooth (enough) boundary $\partial \Omega=\Gamma$. We denote by $n$ be the outer normal to $\Gamma$ and summation over repeated indices is implied and the index that follows a comma represents the partial derivative with respect to the corresponding component of the variable. The indices take values in $\{1, \cdots, d\}$ the summation convention over repeated indices is used.

Below we use $\mathbb{S}^{d}$ to denote the space of second order symmetric tensors on $\mathbb{R}^{d}$ while "." and $\|\cdot\|$ will denote the inner product and the Euclidean norm on $\mathbb{S}^{d}$ and $\mathbb{R}^{d}$, that is

$$
\begin{array}{rlrl}
u . v & =u_{i} v_{i}, & \|v\|=(v . v)^{\frac{1}{2}}, & \forall u, v \in \mathbb{R}^{d}, \\
\sigma . \tau=\sigma_{i j} \tau_{i j}, & \|\tau\|=(\tau . \tau)^{\frac{1}{2}}, & \forall \sigma, \tau \in \mathbb{S}^{d} .
\end{array}
$$

We also use the notations $u_{n}$ and $u_{\tau}$ for the normal and tangential displacement, that is $u_{n}=u . n$ and $u_{\tau}=u-u_{n} n$. Similarly we denote by $\sigma_{n}$ and $\sigma_{\tau}$ the normal and tangential stress tensor given by $\sigma_{n}=\sigma n . n$, $\sigma_{\tau}=\sigma n-\sigma_{n} n$.

We introduce the following functional spaces on $\Omega$;

$$
\begin{aligned}
& H=L^{2}(\Omega)^{d}=\left\{u=\left(u_{i}\right) \mid u_{i} \in L^{2}(\Omega)\right\}, \quad \mathcal{H}=\left\{\sigma=\sigma_{i j}, \sigma_{i j}=\sigma_{j i} \in L^{2}(\Omega)\right\}, \\
& H_{1}=\{u \in H \mid \epsilon(u) \in \mathcal{H}\}, \quad \mathcal{H}_{1}=\{\sigma \in \mathcal{H} \mid \operatorname{Div} \sigma \in H\}
\end{aligned}
$$

endowed with the inner products

$$
\begin{aligned}
& (u, v)_{H}=\int_{\Omega} u_{i} v_{i} d x, \quad(\sigma, \tau)_{\mathcal{H}}=\int_{\Omega} \sigma_{i j} \tau_{i j} d x . \\
& (u, v)_{H_{1}}=(u, v)_{H}+(\varepsilon(u), \varepsilon(v))_{\mathcal{H}}, \quad(\sigma, \tau)_{\mathcal{H}_{1}}=(\sigma, \tau)_{\mathcal{H}}+(\operatorname{Div} \sigma, \text { Div } \tau)_{H} .
\end{aligned}
$$


The associated norms on the spaces $H, \mathcal{H}, H_{1}$ and $\mathcal{H}_{1}$ are denoted by $\|\cdot\|_{H},\|\cdot\|_{\mathcal{H}},\|\cdot\|_{H_{1}}$ and $\|\cdot\|_{\mathcal{H}_{1}}$ respectively.

We recall the well known Green's formula

$$
(\sigma, \varepsilon(v))_{\mathcal{H}}+(\operatorname{Div} \sigma, v)_{H}=\int_{\Gamma} \sigma n . v d a \forall v \in H_{1}
$$

where $\operatorname{Div} \sigma=\left(\sigma_{i j, j}\right)$ and for more details of this formula see [24].

In addition we shall use the following notations. $u$ is the displacement field, $\varepsilon(u)=\left(\varepsilon_{i j}(u)\right)$ to denote the strain tensor, given by $\varepsilon_{i j}(u)=\frac{1}{2}\left(u_{i, j}+u_{j, i}\right)$ and $\sigma=\left(\sigma_{i j}\right)$ being the stress tensor. Lets $\varphi$ denote the electric potential, $E(\varphi)=\left(E_{i}(\varphi)\right)$ is the electric field, which is defined by $E_{i}(\varphi)=-\varphi_{, i}$ and $D=\left(D_{i}\right)$ is the electric displacement field.

The equilibrium equations are given by

$$
\begin{array}{r}
-\operatorname{Div}(\sigma)=f_{0} \text { in } \Omega, \\
\operatorname{div}(D)=q_{0} \text { in } \Omega,
\end{array}
$$

where the constitutive relations for the piezoelectric material are:

$$
\begin{gathered}
\sigma=\mathcal{A} \varepsilon(u)-\mathcal{B}^{*} E(\varphi) \text { in } \Omega, \\
D=\mathcal{B} \varepsilon(u)+\beta E(\varphi) \text { in } \Omega,
\end{gathered}
$$

where $\mathcal{A}=\left(a_{i j k l}\right)$ is a (fourth-order) elasticity tensor, $\mathcal{B}=\left(b_{i j k}\right)$ is the (third-order) piezoelectric tensor, $\mathcal{B}^{*}$ is the transpose of $\mathcal{B}$ and $\beta=\left(\beta_{i j}\right)$ is the electric permitivity and $\operatorname{div}(D)=D_{i, i}$ (see [26]).

To give the mechanical and electrical boundary conditions, we subdivide $\Gamma$ into three disjoints measurable parts $\Gamma_{1}, \Gamma_{2}, \Gamma_{3}$ such that meas $\left(\Gamma_{1}\right)>0$. The body is assumed to be clamped on $\Gamma_{1}$ and surfaces traction of density $f_{2}$ act on $\Gamma_{2}$, on $\Gamma_{3}$ the body can reaches a frictional contact with the so called foundation (insulating foundation). A second partition of $\Gamma$, that is $\Gamma=\Gamma_{3} \cup \Gamma_{a} \cup \Gamma_{b}$. Surface electric charge of density $q_{2}$ acts on $\Gamma_{b}$, and the electric potential vanishes on $\Gamma_{b}$. We use the same symbol $v$ for the trace of $v$ on $\Gamma$.

$$
\begin{aligned}
u & =0 \text { on } \Gamma_{1} . \\
\sigma n & =f_{2} \text { on } \Gamma_{2} \\
\varphi & =0 \text { on } \Gamma_{a} . \\
D . n & =q_{2} \text { on } \Gamma_{b} .
\end{aligned}
$$

The contact and the Coulomb friction conditions:

$$
\begin{gathered}
u_{n}-g \leq 0, \sigma_{n} \leq 0 \text { and } \sigma_{n}\left(u_{n}-g\right)=0 \text { on } \Gamma_{3}, \\
\left\{\begin{array}{l}
\text { If } u_{\tau}=0 \text { then }\left\|\sigma_{\tau}(u)\right\| \leq-\mathcal{F} \sigma_{n}(u) \text { on } \Gamma_{3}, \\
\text { If } u_{\tau} \neq 0 \text { then } \sigma_{\tau}(u)=\mathcal{F} \sigma_{n}(u) \frac{u_{\tau}}{\left\|u_{\tau}\right\|} \text { on } \Gamma_{3},
\end{array}\right.
\end{gathered}
$$

where $\mathcal{F}$ is the friction coefficient and $g$ is the gap between the body and the rigid foundation.

The electric contact condition is;

$$
D . n=0 \text { on } \Gamma_{3} \text {. }
$$

To resume, we consider the following problem:

Problem 1. Find the displacement field $u: \Omega \longrightarrow \mathbb{R}^{d}$ and the electric potential field $\varphi: \Omega \longrightarrow \mathbb{R}$ such that (2)-(12) hold.

To study of Problem 1 we will assume, under Einstein summation convention, that: 


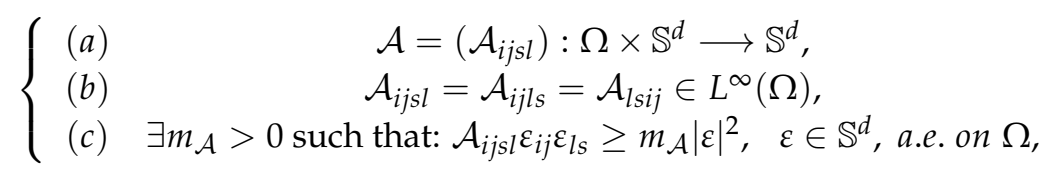

$$
\left\{\begin{array}{c}
\left(\text { a) } \mathcal{B}=\left(\mathcal{B}_{i j k}\right): \Omega \times \mathbb{S}^{d} \longrightarrow \mathbb{R}^{d},\right. \\
\left(\text { b) } \mathcal{B}_{i j k}=\mathcal{B}_{i k j} \in L^{\infty}(\Omega)\right.
\end{array}\right.
$$

$$
\begin{aligned}
& \left\{\begin{array}{cc}
(a) & \beta=\left(\beta_{i j}\right): \Omega \times \mathbb{R}^{d} \longrightarrow \mathbb{R}^{d}, \\
(b) & \beta_{i j}=\beta_{j i} \in L^{\infty}(\Omega),
\end{array}\right. \\
& \text { (c) } \exists m_{\beta}>0 \text { such that: } \beta_{i j} E_{i} E_{j} \geq m_{\beta}|E|^{2}, E \in \mathbb{R}^{d} \text {, a.e. on } \Omega \text {, } \\
& \begin{array}{ll}
f_{0} \in L^{2}(\Omega)^{d}, & f_{2} \in L^{2}\left(\Gamma_{2}\right)^{d}, \\
q_{0} \in L^{2}(\Omega), & q_{2} \in L^{2}\left(\Gamma_{b}\right) .
\end{array}
\end{aligned}
$$

Let us introduce the following Hilbert spaces:

$$
\begin{gathered}
V=\left\{v \in\left[H^{1}(\Omega)\right]^{d} / v=0 \text { on } \Gamma_{1}\right\}, \\
W=\left\{\varphi \in H^{1}(\Omega) / \varphi=0 \text { on } \Gamma_{a}\right\}, \\
K=\left\{v \in\left[H^{\frac{1}{2}}\left(\Gamma_{3}\right)\right]^{d} / v_{n} \leq g \text { on } \Gamma_{3}\right\} .
\end{gathered}
$$

If $u$ and $\varphi$ are regular functions which satisfy (2)-(10), then we find:

$$
\begin{gathered}
\int_{\Omega} \mathcal{A} \varepsilon(u) \varepsilon(v) d x+\int_{\Omega} \mathcal{B}^{*} \nabla \varphi \varepsilon(v) d x=\int_{\Omega} f_{0} v d x+\int_{\Gamma_{2}} f_{2} v d \Gamma+\int_{\Gamma_{3}}(\sigma n) \cdot v d \Gamma \\
-\int_{\Omega} \mathcal{B} \varepsilon(u) \nabla \psi d x+\int_{\Omega} \beta \nabla \varphi \nabla \psi d x=\int_{\Omega} q_{0} \psi d x-\int_{\Gamma_{b}} q_{2} \psi d \Gamma .
\end{gathered}
$$

Let us introduce the functional space $\tilde{V}=V \times W$, which is the Hilbert space endowed with the inner product:

$(\tilde{u}, \tilde{v})_{\tilde{V}}=(u, v)_{V}+(\varphi, \psi)_{W}$ where $\tilde{u}=(u, \varphi), \tilde{v}=(v, \psi) \in \tilde{V}$. Let $a: \tilde{V} \times \tilde{V} \longrightarrow \mathbb{R}$ be the bi-linear form given by:

$$
a(\tilde{u}, \tilde{v})=\int_{\Omega} \mathcal{A} \varepsilon(u) \varepsilon(v) d x+\int_{\Omega} \mathcal{B}^{*} \nabla \varphi \varepsilon(v) d x-\int_{\Omega} \mathcal{B} \varepsilon(u) \nabla \psi d x+\int_{\Omega} \beta \nabla \varphi \nabla \psi d x .
$$

Moreover, by Riesz's representation theorem, we define $\tilde{f} \in \tilde{V}$ by:

$$
(\tilde{f}, \tilde{v})_{\tilde{V}}:=\int_{\Omega} f_{0} v d x+\int_{\Gamma_{2}} f_{2} v d \Gamma+\int_{\Omega} q_{0} \psi d x-\int_{\Gamma_{b}} q_{2} \psi d \Gamma .
$$

Using the previous tools, we find:

$$
a(\tilde{u}, \tilde{v})=(\tilde{f}, \tilde{v})_{\tilde{V}}+\int_{\Gamma_{3}}(\sigma n) \cdot v d \Gamma
$$

Since $(\sigma n) \cdot v=\sigma_{\tau} v_{\tau}+\sigma_{n} v_{n}$, then:

$$
a(\tilde{u}, \tilde{v})=(\tilde{f}, \tilde{v})_{\tilde{V}}+\int_{\Gamma_{3}} \sigma_{\tau} v_{\tau}+\sigma_{n} v_{n} d \Gamma
$$

Let $H_{\Gamma}^{*}$ be the dual space of the space $H_{\Gamma}=\left[H^{\frac{1}{2}}\left(\Gamma_{3}\right)\right]^{d}$ and let us define

$$
\begin{gathered}
M_{T}(\lambda)=\left\{\delta \in H_{\Gamma}^{*}, \quad\left\langle\delta, v_{\tau}\right\rangle_{H_{\Gamma}^{*}, H_{\Gamma}} \leq \int_{\Gamma_{3}} \lambda\left|v_{\tau}\right| d \Gamma, v_{\tau} \in H_{\Gamma}\right\}, \\
M_{N}=\left\{\delta \in H_{\Gamma}^{*}, \quad\langle\delta, v\rangle_{\Gamma_{3}} \leq 0, v \in K_{n}\right\},
\end{gathered}
$$


where $\langle\cdot, \cdot\rangle_{H_{\Gamma}^{*}, H_{\Gamma}}$ denotes the duality product between $H_{\Gamma}^{*}$ and $H_{\Gamma}, K_{n}$ the set of the normal component of admissible displacement, i.e. $K_{n}=\left\{v_{n}, v_{n} \leq g\right\}$.

It is straightforward that $M_{T, N}$ are two closed convex sets of $H_{\Gamma}^{*}$ and $0_{H_{\Gamma}^{*}} \in M_{N, T}$. We introduce two dual Lagrange multipliers $\lambda_{N}$ and $\lambda_{T} \in M$ as follows:

$$
\left\langle\lambda_{N}, v\right\rangle_{\Gamma_{3}}:=-\int_{\Gamma_{3}} \sigma_{n} v_{n} d \Gamma, \quad v \in V, \text { and }\left\langle\lambda_{T}, v\right\rangle_{\Gamma_{3}}:=-\int_{\Gamma_{3}} \sigma_{\tau} v_{\tau} d \Gamma, \quad v \in V .
$$

We define two bi-linear and continuous forms $b_{1}$ and $b_{2}$ for all $v \in V, \delta_{1}, \delta_{2} \in H_{\Gamma}^{*}$ as follows:

$$
\begin{array}{ll}
b_{1}: \tilde{V} \times H_{\Gamma}^{*} \longrightarrow \mathbb{R}, & b_{1}\left(\tilde{v}, \delta_{1}\right):=\left\langle\delta_{1}, v\right\rangle_{\Gamma_{3}}, \\
b_{2}: \tilde{V} \times H_{\Gamma}^{*} \longrightarrow \mathbb{R}, & b_{2}\left(\tilde{v}, \delta_{2}\right):=\left\langle\delta_{2}, v\right\rangle_{\Gamma_{3}} .
\end{array}
$$

We see that $b_{1}\left(\tilde{u}, \lambda_{N}\right)=-\int_{\Gamma_{3}} \sigma_{n} u_{n} d \Gamma$, and by definition of $M_{N}$ we have:

$$
b_{1}\left(\tilde{u}, \delta_{1}-\lambda_{N}\right) \leq 0, \quad \forall \delta_{1} \in M_{N} .
$$

Also, taking into account the definition of $M_{T}, \lambda_{T}$, and the assumption (10), we have:

$$
b_{2}\left(\tilde{u}, \lambda_{T}\right)=\left\langle\lambda_{T}, u\right\rangle_{\Gamma_{3}}=-\int_{\Gamma_{3}} \sigma_{\tau} u_{\tau} d \Gamma
$$

Keeping in mind that the Sobolev trace operator is linear and continuous, it is clear that there exists $M_{b_{i}}>0$ such that:

$$
\left|b_{i}\left(\tilde{v}, \delta_{i}\right)\right| \leq M_{b_{i}}|| \tilde{v}||_{\tilde{v}}|| \delta_{i} \|_{H_{\Gamma}^{*},} i=1,2
$$

In addition, using the properties of the Sobolev trace operator it can be shown that there exists $\alpha_{i}>0$ such that:

$$
\inf _{\delta_{i} \in H_{\Gamma}^{*} \backslash\{0\}} \sup _{\tilde{v} \neq 0} \frac{b_{i}\left(\tilde{v}, \delta_{i}\right)}{\|\tilde{v}\|_{\tilde{V}}\left\|\delta_{i}\right\|_{H_{\Gamma}^{*}}} \geq \alpha_{i}, i=1,2 .
$$

The following weak formulation of Problem 1 is then obtained :

Problem 2. (Weak formulation of Problem Problem 1) Find $\tilde{u} \in \tilde{V}$ and $\lambda=\left(\lambda_{N}, \lambda_{T}\right) \in M_{N} \times M_{T}\left(\mathcal{F} \lambda_{N}\right)$ such that:

$$
\begin{array}{cc}
a(\tilde{u}, \tilde{v})+b_{1}\left(\tilde{v}, \lambda_{N}\right)+b_{2}\left(\tilde{v}, \lambda_{T}\right)=(\tilde{f}, \tilde{v})_{\tilde{V}}, \quad \forall \tilde{v} \in \tilde{V}, \\
b_{1}\left(\tilde{u}, \delta_{1}-\lambda_{N}\right) \leq 0, & \forall \delta_{1} \in M_{N}, \\
b_{2}\left(\tilde{u}, \delta_{2}-\lambda_{T}\right) \leq 0, \quad \forall \delta_{2} \in M_{T}\left(\mathcal{F} \lambda_{N}\right) .
\end{array}
$$

\subsection{Main results}

In this section we present our main results.

Theorem 1. Assume (13)-(17), then the Problem 2 has unique solution $(\tilde{u}, \lambda) \in \tilde{V} \times M$. Moreover if $\left(\tilde{u}_{1}, \lambda\right)$ and $\left(\tilde{u}_{2}, \beta\right)$ are two solutions of Problem 2 for given data $\tilde{f}_{1}$ and $\tilde{f}_{2}$ respectively, then

$$
\left\|\tilde{u}_{1}-\tilde{u}_{2}\right\|_{\tilde{V}}+\|\lambda-\beta\|_{H_{\Gamma}^{*} \times H_{\Gamma}^{*}} \leq C\left(\left\|\tilde{f}_{1}-\tilde{f}_{2}\right\|\right) .
$$

We denote $b_{1}\left(v, \delta_{1}\right)+b_{2}\left(v, \delta_{2}\right)=\left\langle\delta_{1}, v\right\rangle_{\Gamma_{3}}+\left\langle\delta_{2}, v\right\rangle_{\Gamma_{3}}=\left\langle\delta_{1}+\delta_{2}, v\right\rangle_{\Gamma_{3}}$, hence there exists $\alpha>0$ such that

$$
\inf _{\delta \in H_{\Gamma}^{*} \backslash\{0\}} \sup _{v \neq 0} \frac{b(v, \delta)}{\|v\|_{V}\|\delta\|_{H_{\Gamma}^{*}}} \geq \alpha
$$

where $b(\cdot, \cdot)=b_{1}(\cdot, \cdot)+b_{2}(\cdot, \cdot): \tilde{V} \times M_{N} \times M_{T} \longrightarrow \mathbb{R}$. Now we introduce a numerical scheme to get numerically the solution of the Problem 2 . The scheme is an fixed point iterative and is stated in the following Algorithm 1. 


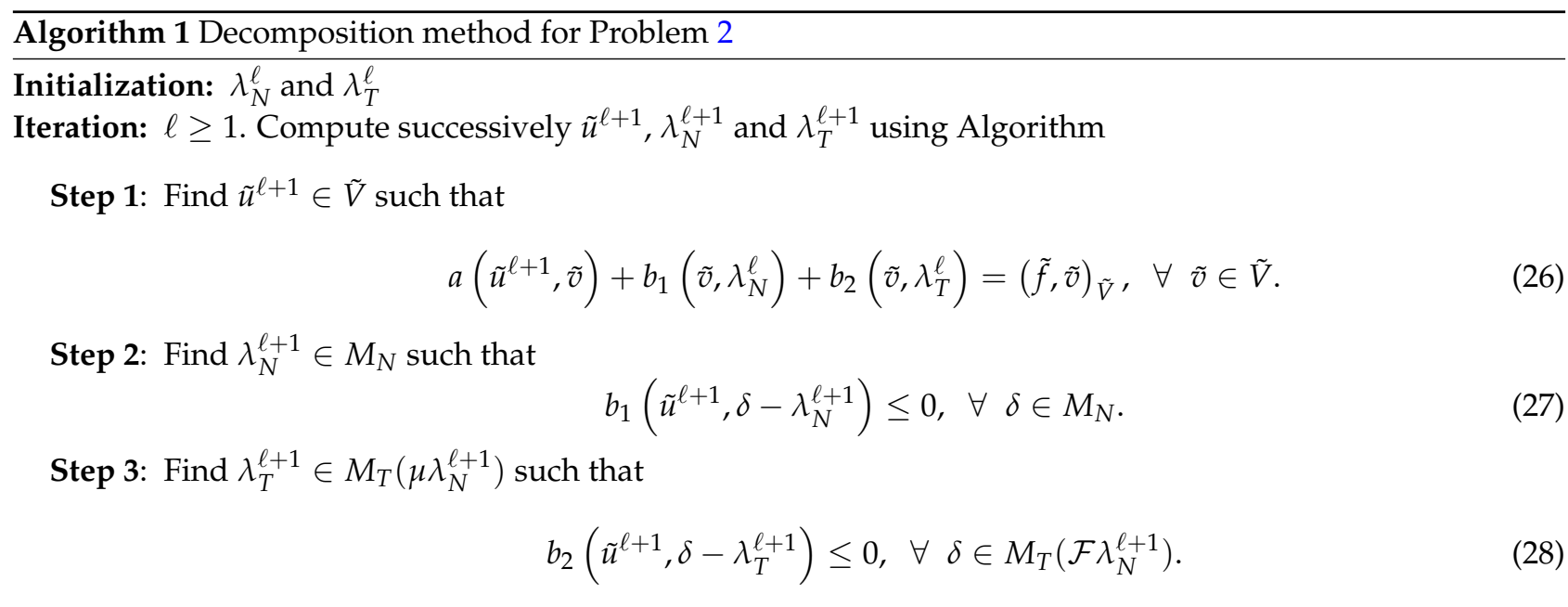

Proposition 1. Let $\left(u^{\ell}, \lambda^{\ell}\right)$ be the solution generated by the Algorithm 1, then

$$
\left\|u^{\ell}-u\right\|_{\tilde{V}}+\left\|\lambda^{\ell}-\lambda\right\|_{H_{\Gamma}^{*} \times H_{\Gamma}^{*}} \longrightarrow 0, \text { as } \ell \longrightarrow+\infty .
$$

The proof of the main results will be presented in the next section.

\section{Proof of the main result}

Let $X$ and $Y$ two Hilbert spaces endowed with the inner product $(\cdot, \cdot)_{X}$ and $(\cdot, \cdot)_{Y}$ respectively and let us consider two bi-linear forms as follows:

$a(\cdot, \cdot): X \times X \longrightarrow \mathbb{R}$, generally non symmetric, such that

$$
\begin{gathered}
\exists M_{a}>0 \text { such that }|a(u, v)| \leq M_{a}\|u\|_{X} \|_{\mid v \|_{X},} \quad \forall u, v \in X, \\
\exists m_{a}>0 \text { such that } a(v, v) \geq m_{a}\|v\|_{X}^{2}, \quad \forall v \in X,
\end{gathered}
$$

and $b(\cdot, \cdot): X \times Y \times Y \longrightarrow \mathbb{R}, b(v, \lambda)=b_{1}\left(v, \lambda_{N}\right)+b_{2}\left(v, \lambda_{T}\right)$ such that

$$
\exists M_{b}>0 \text { such that }|b(v, \delta)| \leq M_{b}\|v\|_{X}\|\delta\|_{Y \times Y}, \quad \forall \quad(v, \delta) \in X \times Y \times Y,
$$

$\exists M_{b_{i}}>0$ such that

$$
\left|b_{i}(v, \delta)\right| \leq M_{b_{i}}\|v\|_{X}\|\delta\|_{Y \times Y}, \quad \forall \quad(v, \delta) \in X \times Y \times Y, i=1,2,
$$

there exists $\alpha>0$ such that

$$
\inf _{\delta \in Y \times Y \backslash\{0\}} \sup _{v \in X \backslash\{0\}} \frac{b(v, \delta)}{\|v\|_{X}\|\delta\|_{Y \times Y}} \geq \alpha .
$$
problem:

Now, let $M=M_{N} \times M_{T} \subset Y \times Y$ be closed and convex set that contain $0_{Y \times Y}$, we consider the following

Problem 3. For given $f \in X$, find $u \in X$ and $\lambda=\left(\lambda_{N}, \lambda_{T}\right) \in M$ such that:

$$
\begin{array}{rr}
a(u, v)+b(v, \lambda)=(f, v)_{X}, & \forall v \in X, \\
b_{1}\left(u, \delta-\lambda_{N}\right) \leq 0, & \forall \delta \in M_{N}, \\
b_{2}\left(u, \delta-\lambda_{T}\right) \leq 0, & \forall \delta \in M_{T} .
\end{array}
$$

We have the following result; 
Theorem 2. Let $f \in X$ and assume that (30)-(34) hold. Then, there exists a unique solution $(u, \lambda)$ of Problem AWF Moreover, if $\left(u_{1}, \lambda\right)$ and $\left(u_{2}, \gamma\right)$ are two solutions of the Problem AWF for given data functions $f_{1} \in X$ and $f_{2} \in X$ respectively, then, there exists $S C>0$ such that:

$$
\left\|u_{1}-u_{2}\right\|_{X}+\|\lambda-\beta\|_{Y \times Y} \leq K\left(\left\|f_{1}-f_{2}\right\|_{X}\right) .
$$

Proof. We consider the symmetric $a_{0}(.,$.$) and anti-symmetric c(.,$.$) part of a(.,$.$) respectively, defined by$

$$
\begin{array}{cc}
a_{0}: X \times X \longrightarrow \mathbb{R}, a_{0}(u, v):=(a(u, v)+a(v, u)) / 2, & u, \forall v \in X, \\
c: X \times X \longrightarrow \mathbb{R}, c(u, v):=(a(u, v)-a(v, u)) / 2, & u, \forall v \in X .
\end{array}
$$

For given $0<t<1$, let us present the following bi-linear form

$$
a_{t}: X \times X \longrightarrow \mathbb{R}, a_{t}(u, v):=a_{0}(u, v)+t c(u, v), \quad \forall u, v \in X .
$$

For all $t \in[0,1]$, we note that

$$
a_{t}(v, v) \geq m_{a}\left|\|v\|_{X}^{2}, \quad\right| a_{t}(u, v) \mid \leq 2 M_{a}\|u\|_{X}\|v\|_{X}, \quad \forall u, v \in X .
$$

Let us consider the following auxiliary perturbed problem:

Problem 4. (Auxiliary perturbed problem) For given $f \in X$, find $u \in X$ and $\lambda \in M$, such that

$$
\begin{array}{rr}
a_{t}(u, v)+b(v, \lambda)=(f, v)_{X}, & \forall v \in_{X}, \\
b_{1}\left(u, \delta-\lambda_{N}\right) \leq 0, & \forall \delta \in M_{N}, \\
b_{2}\left(u, \delta-\lambda_{T}\right) \leq 0, & \forall \delta \in M_{T} .
\end{array}
$$

The rest of the proof will be treated by steps.

Step 1. If $t=0$, the Problem 4 has unique solution. Indeed, if $l=0$ the problem is equivalent to the saddle point problem: find $u \in X$ and $\lambda \in M$ such that

$$
\mathcal{L}(u, \delta) \leq \mathcal{L}(u, \lambda) \leq \mathcal{L}(v, \lambda), \quad \forall v \in X, \delta \in M,
$$

where $\mathcal{L}: X \times M \longrightarrow \mathbb{R}$ is defined by:

$$
\begin{aligned}
\mathcal{L}(v, \delta) & := & \frac{1}{2} a_{0}(v, v)-(f, v)_{X}+b_{2}\left(v, \delta_{2}\right)+b_{1}\left(v, \delta_{1}\right) \\
& = & \frac{1}{2} a_{0}(v, v)-(f, v)_{X}+b(v, \delta),
\end{aligned}
$$

$\mathcal{L}(.,$.$) has at least one solution, see [10], in fact, from \mathcal{L}(v, 0)=\frac{1}{2} a_{0}(v, v)-(f, v)_{X}$ and the coercivity of $a_{0}(.,$. we have

$$
\lim _{\|v\|_{X} \rightarrow+\infty} \mathcal{L}(v, 0)=+\infty
$$

Moreover

$$
\lim _{\|\delta\|_{Y \times Y} \rightarrow+\infty} \inf _{v \in X} \mathcal{L}(v, \delta)=-\infty .
$$

Indeed, let $\delta_{0}$ be an element of $M$ and let $u_{\delta_{0}} \in X$ be the solution of the equation

$$
a_{0}\left(u_{\delta_{0}}, v\right)+b\left(v, \delta_{0}\right)=(f, v)_{X,} \quad \forall v \in X,
$$

which is equivalent that $u_{\delta_{0}}$ is the solution of the following minimization problem

$$
\inf _{v \in X} \mathcal{L}\left(v, \delta_{0}\right)=\frac{1}{2} a_{0}(v, v)-(f, v)_{X}+b\left(v, \delta_{0}\right),
$$


that is

$$
\frac{1}{2} a_{0}\left(u_{\delta_{0}}, u_{\delta_{0}}\right)-\left(f, u_{\delta_{0}}\right) \mathrm{X}+b\left(u_{\delta_{0}}, \delta_{0}\right)=\inf _{v \in X} \mathcal{L}\left(v, \delta_{0}\right) .
$$

Substituting $v=u_{\delta_{0}}$ in (44), we get

$$
\frac{1}{2} a_{0}\left(u_{\delta_{0}}, u_{\delta_{0}}\right)-\left(f, u_{\delta_{0}}\right) X+b\left(u_{\delta_{0}}, \delta_{0}\right)=-\frac{1}{2} a\left(u_{\delta_{0}}, u_{\delta_{0}}\right),
$$

which implies that

$$
\inf _{v \in X} \mathcal{L}\left(v, \delta_{0}\right) \leq \frac{-m_{a}}{2}\left\|u_{\delta_{0}}\right\|_{X}^{2}
$$

Additionally, using the inf-sup property of the form $b(. .$.$) ; we deduce that there exists a constant C>0$ such that

$$
\left\|\delta_{0}\right\|_{Y \times Y} \leq C\left(\|f\|_{X}+\left\|u_{\delta_{0}}\right\|_{X}\right) .
$$

From (45) we deduce (38), which implies the existence of solution of Problem 4.

To show the uniqueness of the solution, let us assumes that $\left(u_{1}, \lambda\right)$ and $\left(u_{2}, \gamma\right)$ are two solutions of the problem

$$
\begin{array}{ll}
a_{0}\left(u_{1}, v\right)+b(v, \lambda)=(f, v)_{X}, \quad & \forall v \in X, \\
a_{0}\left(u_{2}, v\right)+b(v, \gamma)=(f, v)_{X}, & \forall v \in X .
\end{array}
$$

By subtracting these two equations, we find

$$
a_{0}\left(u_{1}-u_{2}, v\right)+b(v, \lambda)-b(v, \gamma)=0
$$

If we set $v=u_{1}-u_{2}$, we get $a_{0}\left(u_{1}-u_{2}, u_{1}-u_{2}\right)+b\left(u_{1}-u_{2}, \lambda\right)-b\left(u_{1}-u_{2}, \gamma\right)=0$,

$$
\begin{aligned}
a_{0}\left(u_{1}-u_{2}, u_{1}-u_{2}\right) & =-b\left(u_{1}-u_{2}, \lambda\right)+b\left(u_{1}-u_{2}, \gamma\right) \\
& =b\left(u_{2}-u_{1}, \lambda\right)+b\left(u_{1}-u_{2}, \gamma\right) \\
& =b_{1}\left(u_{2}-u_{1}, \lambda_{N}\right)+b_{1}\left(u_{2}-u_{1}, \lambda_{T}\right)+b_{2}\left(u_{1}-u_{2}, \gamma_{1}\right)+b_{2}\left(u_{1}-u_{2}, \gamma_{2}\right) \\
& =b_{1}\left(u_{2}-u_{1}, \lambda_{1}-\gamma_{1}\right)+b_{2}\left(u_{2}-u_{1}, \lambda_{T}-\gamma_{2}\right) \leq 0
\end{aligned}
$$

and by coercivity of $a_{0}$, we have $u_{1}=u_{2}$. Moreover

$$
0=-a_{0}\left(u_{1}-u_{2}, v\right)=b(v, \lambda-\gamma)
$$

and by inf-sup property of $b(.,$.$) , we have$

$$
\alpha\|\lambda-\gamma\|_{Y \times Y} \leq \sup _{v \in X} \frac{b(v, \lambda-\gamma)}{\|v\|_{X}}=0,
$$

and finally $\lambda=\gamma$.

Step 2. Assume now that $f \in X$, there exists a unique solution $(u, \lambda) \in X \times M$ of the Problem 4 , when we have two solutions $\left(u_{1}, \lambda\right)$ and $\left(u_{2}, \gamma\right)$ of Problem 4 corresponding to two given data $f_{1} \in X \times X$ and $f_{2} \in X \times X$ respectively, then

$$
\left\|u_{1}-u_{2}\right\|_{X}+\|\lambda-\gamma\|_{Y \times Y} \leq \frac{\alpha+m_{a}+2 M_{a}}{\alpha m_{a}}\left\|f_{1}-f_{2}\right\|_{X} .
$$

In fact

$$
\begin{gathered}
a_{t}\left(u_{1}-u_{2}, u_{1}-u_{2}\right)=\left(f_{1}-f_{2}, u_{1}-u_{2}\right)_{X}+b\left(u_{1}-u_{2}, \gamma-\lambda\right), \\
b_{1}\left(u_{1}-u_{2}, \gamma_{1}-\lambda_{N}\right) \leq 0, \\
b_{2}\left(u_{1}-u_{2}, \gamma_{2}-\lambda_{T}\right) \leq 0 .
\end{gathered}
$$

Since $a_{t}$ is coercive and, hence

$$
\left\|u_{1}-u_{2}\right\|_{X} \leq \frac{1}{m_{a}}\left\|f_{1}-f_{2}\right\|_{X}
$$


In addition, $b(v, \lambda-\gamma)=\left(f_{1}-f_{2}, v\right)_{X}+a_{r}\left(u_{2}-u_{1}, v\right)$ and by inf-sup property of $b(.,$.$) we have$

$$
\alpha\|\lambda-\gamma\|_{Y \times Y} \leq \sup _{v \in X \backslash\{0\}} \frac{b(v, \lambda-\gamma)}{\|v\|_{X}} \leq\left\|f_{1}-f_{2}\right\|_{X}+2 M_{a}\left\|u_{1}-u_{2}\right\|_{X}
$$

that is

$$
\|\lambda-\gamma\|_{Y \times Y} \leq \frac{m_{a}+2 M_{a}}{\alpha m_{a}}\left\|f_{1}-f_{2}\right\|_{X}
$$

Hence (47) and (48) lead to

$$
\left\|u_{1}-u_{2}\right\|_{X}+\|\lambda-\gamma\|_{Y \times Y} \leq \frac{\alpha+m_{a}+2 M_{a}}{\alpha m_{a}}\left\|f_{1}-f_{2}\right\|_{X} .
$$

Step 3. Let $\tau \in[0,1]$. Assume for given $f, g \in X$ there exists a unique solution of Problem 4 with $t=\tau$, $(u, \lambda) \in X \times M$. Then for given $f \in X$ there exists a unique solution $(u, \lambda) \in X \times M$ of Problem 4 with $t \in\left[\tau ; \tau+t_{0}\right] \subset[0,1]$, where:

$$
0<t_{0}<\frac{\alpha m_{a}}{M_{a}\left(\alpha+m_{a}+2 M_{a}\right)}<1
$$

Indeed, given $f \in X$, we define the operator $T: X \times M \longrightarrow X \times M$ as follows $T(w, \xi):=(u, \lambda)$ if $(u, \lambda)$ is the solution of the following problem:

Problem 5. For given $f \in X$, find $u \in X$ and $\lambda \in M$, such that

$$
\begin{aligned}
a_{\tau}(u, v)+b(v, \lambda)=\left(F_{s}, v\right)_{X}, & \forall v \in X, \\
b_{1}\left(u, \delta-\lambda_{N}\right) \leq 0, & \forall \delta \in M_{N}, \\
b_{2}\left(u, \delta-\lambda_{T}\right) \leq 0, & \forall \delta \in M_{T},
\end{aligned}
$$

where $\left(F_{s}, v\right)_{X}=(f, v)_{X}-(s-\tau) c(w, v), \quad \tau \leq s \leq \tau+t_{0} \leq 1$

We will show that $T$ is a contraction. To this end, we consider two pairs $\left(w_{1}, \xi\right)$ and $\left(w_{2}, \chi\right) \in X \times Y \times Y$. We have

$$
\left\|T\left(w_{1}, \xi\right)-T\left(w_{2}, \chi\right)\right\|_{X \times Y \times Y}=\left\|u_{1}-u_{2}\right\|_{X}+\|\lambda-\beta\|_{Y \times Y} .
$$

By the same argument in (48) and by definition of $F_{s}$

$$
\|\lambda-\gamma\|_{Y \times Y} \leq \frac{m_{a}+2 M_{a}}{\alpha m_{a}} t_{0} M_{a}|| w_{1}-w_{2} \|_{X} .
$$

In addition,

$$
\left\|u_{1}-u_{2}\right\|_{X} \leq \frac{1}{m_{a}} t_{0} M_{a}\left\|w_{1}-w_{2}\right\|_{X}
$$

and hence,

$$
\begin{gathered}
\left\|u_{1}-u_{2}\right\|_{X}+\|\lambda-\gamma\|_{Y \times Y} \leq \frac{t_{0} M_{a}\left(\alpha+m_{a}+2 M_{a}\right)}{\alpha m_{a}}\left\|w_{1}-w_{2}\right\|_{X}, \\
\left\|u_{1}-u_{2}\right\|_{X}+\|\lambda-\gamma\|_{Y \times Y} \leq \frac{t_{0} M_{a}\left(\alpha+m_{a}+2 M_{a}\right)}{\alpha m_{a}}\left\|\left(w_{1}, \xi\right)-\left(w_{2}, \chi\right)\right\|_{X \times Y \times Y,}
\end{gathered}
$$

which implies that $T$ is a contraction and by Banach fixed theorem we conclude that $T$ has unique fixed point. Let $(\bar{u}, \bar{\lambda})$ be the unique fixed point of $T$, using the definition of the operator $T$, we deduce that

$$
\begin{aligned}
a_{\tau}(\bar{u}, v)+b(v, \bar{\lambda})=\left(F_{S}, v\right)_{X}, & \forall v \in X, \\
b_{1}\left(\bar{u}, \delta-\bar{\lambda}_{N}\right) \leq 0, & \forall \delta \in M_{N}, \\
b_{2}\left(\bar{u}, \delta-\bar{\lambda}_{T}\right) \leq 0, & \forall \delta \in M_{T}
\end{aligned}
$$

and $\left(F_{s}, v\right)_{X}=(f, v)_{X}-(s-\tau) c(\bar{u}, v)$, for $\tau \leq s \leq \tau+t_{0} \leq 1$. 
We substitute $F_{S}$ in the first equation, we find $(\bar{u}, \bar{\lambda})$ be the unique fixed point of $T$, using the definition of the operator $T$, we deduce that

$$
\begin{aligned}
a_{\tau}(\bar{u}, v)+(s-\tau) c(\bar{u}, v)+b(v, \bar{\lambda})= & (f, v)_{X}, \quad \forall v \in X, \\
& b_{1}\left(\bar{u}, \delta-\bar{\lambda}_{N}\right) \leq 0, \quad \forall \delta \in M_{N}, \\
& b_{2}\left(\bar{u}, \delta-\bar{\lambda}_{N}\right) \leq 0, \quad \forall \delta \in M_{T},
\end{aligned}
$$

that is

$$
\begin{array}{cl}
a_{0}(\bar{u}, v)+s c(\bar{u}, v)+b(v, \bar{\lambda})=a_{s}(\bar{u}, v)+b(v, \bar{\lambda})=(f, v)_{X}, & \forall v \in X, \\
& b_{1}\left(\bar{u}, \delta-\bar{\lambda}_{N}\right) \leq 0, \quad \forall \delta \in M_{N}, \\
& b_{2}\left(\bar{u}, \delta-\bar{\lambda}_{T}\right) \leq 0, \quad \forall \delta \in M_{T},
\end{array}
$$

which gives the existence of solution. In order to justify the uniqueness, let us assume that the problem with $l=s \in\left[\tau, \tau+t_{0}\right]$ has two solutions $\left(u_{1}, \lambda\right)$ and $\left(u_{2}, \gamma\right)$, we have

$$
\begin{aligned}
a_{s}\left(u_{1}-u_{2}, v\right)+b(v, \lambda-\gamma) & =0 \\
a_{s}\left(u_{1}-u_{2}, u_{1}-u_{2}\right) & =b\left(u_{2}-u_{1}, \lambda-\gamma\right) \\
& =b_{1}\left(u_{2}-u_{1}, \lambda_{N}-\beta_{1}\right)+b_{2}\left(u_{2}-u_{1}, \lambda_{T}-\gamma_{2}\right) \leq 0
\end{aligned}
$$

hence, by coercivity of $a_{s}$, we get $u_{1}=u_{2}$ and $\lambda=\gamma$.

Step 4. Using Step 3, a finite number of times, we deduce that the Problem 4 admits a unique solution $(u, \lambda)$ for $t=1$.

Step 5. In order to get (44), let us consider the data $f_{1,2} \in X$

$$
\begin{array}{cl}
a\left(u_{1}, v\right)+b(v, \lambda)=\left(f_{1}, v\right)_{X}, & \forall v \in X, \\
b_{1}\left(u_{1}, \delta-\lambda_{N}\right) \leq 0, & \forall \delta \in M_{N}, \\
b_{2}\left(u_{1}, \delta-\lambda_{T}\right) \leq 0, & \forall \delta \in M_{T},
\end{array}
$$

and

$$
\begin{array}{cl}
a\left(u_{2}, v\right)+b(v, \gamma)=\left(f_{2}, v\right)_{X}, & \forall v \in X, \\
b_{1}\left(u_{2}, \delta-\gamma_{1}\right) \leq 0, \quad & \forall \delta \in M_{N}, \\
b_{2}\left(u_{2}, \delta-\gamma_{2}\right) \leq 0, \quad \forall \delta \in M_{T} .
\end{array}
$$

By subtracting this two equations, we find

$$
a\left(u_{1}-u_{2}, v\right)+b(v, \lambda)-b(v, \gamma)=\left(f_{1}-f_{2}, v\right)_{X}
$$

For $v=u_{1}-u_{2}$, we have

$$
a\left(u_{1}-u_{2}, u_{1}-u_{2}\right)+b\left(u_{1}-u_{2}, \lambda\right)-b\left(u_{1}-u_{2}, \gamma\right)=\left(f_{1}-f_{2}, u_{1}-u_{2}\right)_{X},
$$

which implies that

$$
\begin{aligned}
a\left(u_{1}-u_{2}, u_{1}-u_{2}\right) & =b\left(u_{1}-u_{2}, \gamma\right)-b\left(u_{1}-u_{2}, \lambda\right)+\left(f_{1}-f_{2}, u_{1}-u_{2}\right)_{X} \\
& \leq b_{2}\left(u_{1}-u_{2}, \gamma_{2}-\lambda_{T}\right)+\left(f_{1}-f_{2}, u_{1}-u_{2}\right)_{X} \\
m_{a}\left\|u_{1}-u_{2}\right\|_{X}^{2} & \leq\left\|f_{1}-f_{2}\right\|_{X}\left\|u_{1}-u_{2}\right\|_{X}
\end{aligned}
$$

that is

$$
m_{a}\left\|u_{1}-u_{2}\right\|_{X}^{2} \leq\left\|f_{1}-f_{2}\right\|_{X}\left\|u_{1}-u_{2}\right\|_{X}
$$


and by (34), we have

$$
\alpha|| \beta-\lambda\left\|_{Y \times Y} \leq M_{a}|| u_{1}-u_{2}\right\|_{X}+\left\|f_{1}-f_{2}\right\|_{X} .
$$

Using (56), we can write

$$
m_{a}\left\|u_{1}-u_{2}\right\|_{X}^{2} \leq \frac{1}{2 c_{1}}\left\|f_{1}-f_{2}\right\|_{X}^{2}+\frac{c_{1}}{2}\left\|u_{1}-u_{2}\right\|_{X}^{2}+\frac{c_{2}}{2}\|\gamma-\lambda\|_{Y \times Y}^{2},
$$

where $c_{1}, c_{2}$ are strictly positive constants.

By combining this inequality and (57), we deduce that

$$
\left(m_{a}-\frac{c_{1}}{2}-\frac{c_{2} M_{a}^{2}}{\alpha^{2}}\right)\left\|u_{1}-u_{2}\right\|_{X}^{2} \leq\left(\frac{1}{2 c_{1}}+\frac{c_{2}}{\alpha^{2}}\right)\left\|f_{1}-f_{2}\right\|_{X}^{2}
$$

The constants $c_{1}$ and $c_{2}$ are chosen such that $\left(m_{a}-\frac{c_{1}}{2}-\frac{c_{2} M_{a}^{2}}{\alpha^{2}}\right)$, we deduce that there exists $c=$ $c\left(m_{a}, M_{a}, M_{b}, \alpha\right)$ such that

$$
\left\|u_{1}-u_{2}\right\|_{X} \leq c\left(\left\|f_{1}-f_{2}\right\|_{X}\right)
$$

Finally, combining (57) and (60), we have (47).

Proof of Theorem 1. We consider $X=\tilde{V}, Y=H_{\Gamma}^{*}$ and $M_{N} \times M_{N}$ given by (18). The subset $M_{N} \times M_{N}$ is a non-empty, closed, convex of $H_{\Gamma}^{*} \times H_{\Gamma}^{*}$ and $0_{H_{\Gamma}^{*}} \in M$.

By using (12) and (15) we deduce that there exists $M_{a}=M_{a}(\mathcal{A}, \mathcal{E}, \beta)>0$ and $m_{a}=m_{a}(\mathcal{A}, \beta)>0$ such that the bilinear form $a(.,$.$) satisfies$

$$
\begin{gathered}
|a(\tilde{u}, \tilde{v})| \leq M_{a}\|\tilde{u}\|_{V}\|\tilde{v}\|_{\tilde{V}}, \quad \forall \tilde{u}, \tilde{v} \in V, \\
a(\tilde{u}, \tilde{u}) \geq m_{a}\|\tilde{u}\|_{V}^{2}, \quad \forall \tilde{u} \in V .
\end{gathered}
$$

By the conditions (21) and (22) we deduce that the bilinear form $b(.,$.$) satisfies (32). Using inf-sup property$ (34) and Theorem 2 we find the result of Theorem 1.

Proof of Proposition 1. To prove the convergence result 1 of the Algorithm 1, lets reconsider the following perturbed problem, for $l \in\left[\tau ; \tau+t_{0}\right] \subset[0,1]$, where this time:

$$
0<t_{0}<\frac{\alpha m_{a}-\alpha m_{a} M_{b}}{M_{a}\left(\alpha+m_{a}+2 M_{a}\right)}
$$

if $M_{b}<1$. If $M_{b}>1$ we take $l \in\left[\tau+t_{0} ; \tau\right] \subset[0,1]$ with

$$
\frac{-\alpha m_{a} M_{b}}{M_{a}\left(\alpha+m_{a}+2 M_{a}\right)}<t_{0}<\frac{\alpha m_{a}-\alpha m_{a} M_{b}}{M_{a}\left(\alpha+m_{a}+2 M_{a}\right)} .
$$

Given $f, g \in X$, we define the mapping $T: X \times M \longrightarrow X \times M$ as follows $T(w, \xi):=(u, \lambda)$ if $(u, \lambda)$ is the solution of the following fixed point problem:

Problem 6. (Fixed point problem) For given $f, g \in X$, find $u \in X$ and $\lambda \in M$, such that

$$
\begin{array}{cl}
a_{\tau}(u, v)=\left(F_{s}, v\right)_{X}-b(v, \xi), & \forall v \in X, \\
b_{1}\left(u, \delta-\lambda_{N}\right) \leq 0, & \forall \delta \in M_{N}, \\
b_{2}\left(u, \delta-\lambda_{T}\right) \leq 0, & \forall \delta \in M_{T} .
\end{array}
$$

where $\left(F_{s}, v\right)_{X}=(f, v)_{X}-(s-\tau) c(w, v)$ and $\tau \leq s \leq \tau+t_{0} \leq 1$.

It is straightforward that

$$
\left\|u_{1}-u_{2}\right\|_{X}+\|\lambda-\gamma\|_{Y \times Y} \leq \frac{t_{0} M_{a}\left(\alpha+m_{a}+2 M_{a}\right)+\alpha m_{a} M_{b}}{\alpha m_{a}}\left\|\left(w_{1}, \xi\right)-\left(w_{2}, \chi\right)\right\|_{X \times Y \times Y}
$$


By the conditions (61)-(62) and (66), the operator $T$ is a contraction. This implies that there exists $(\bar{u}, \bar{\lambda})$ such that $T(\bar{u}, \bar{\lambda})=(\bar{u}, \bar{\lambda})$. Hence we have $T\left(u^{\ell}, \lambda^{\ell}\right)=\left(u^{\ell+1}, \lambda^{\ell+1}\right)$ and the following scheme converges;

Problem 7. For given $f \in X$, find $u^{\ell+1} \in X$ and $\lambda^{\ell+1} \in M$, such that

$$
\begin{array}{rr}
a_{\tau}\left(u^{\ell+1}, v\right)=\left(F_{s}, v\right)_{X}-b\left(v, \lambda^{\ell}\right), & \forall v \in X, \\
b_{1}\left(u^{\ell+1}, \delta-\lambda_{N}^{\ell+1}\right) \leq 0, & \forall \delta \in M_{N}, \\
b_{2}\left(u^{\ell+1}, \delta-\lambda_{T}^{\ell+1}\right) \leq 0, & \forall \delta \in M_{T} .
\end{array}
$$

The convergence of the iterative fixed point scheme in the Algorithm 1 follows directly.

\section{Discretization and numerics}

The problem is now how to identify the multipliers $\lambda_{N}$ and $\lambda_{T}$ in the convex sets $M_{1,2}$. One manner to do this, is the use of Projection maps. To this end, we consider the finite dimensional spaces $V^{h} \subset V, K^{h}=K \cap V^{h}$ and $W^{h} \subset W$ approximating the spaces $V$ and $W$, respectively, in which $h>0$ denotes the spatial discretization parameter. Let us define:

$$
X_{n}^{h}=\left\{v_{n \mid \Gamma_{3}}^{h}: v^{h} \in V^{h}\right\}, X_{T}^{h}=\left\{v_{\tau \mid \Gamma_{3}}^{h}: v^{h} \in V^{h}\right\},
$$

and

$$
X^{h}=\left\{v_{\mid \Gamma_{3}}^{h}: v^{h} \in V^{h}\right\}=X_{n}^{h} \times X_{T}^{h} .
$$

Let us denote also $X_{n}^{* h} \subset X_{n}^{*} \cap L^{2}\left(\Gamma_{3}\right)$ and $X_{T}^{* h} \subset X_{T}^{*} \cap L^{2}\left(\Gamma_{3} ; \mathbb{R}^{d-1}\right)$ the finite discretizations of $X_{n}^{*}$ and $X_{T}^{*}$ respectively, such that the following discrete Babuska-Brezzi inf-sup conditions hold;

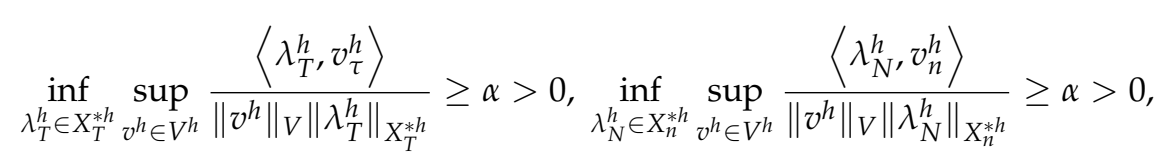

with $\alpha$ independent of $h$.

We consider the following discrete approximation of Problem 2:

Problem 8. Find $\tilde{u}^{h} \in \tilde{V}^{h}$ and $\lambda^{h}=\left(\lambda_{N}^{h}, \lambda_{T}^{h}\right) \in M_{N}^{h} \times M_{T}^{h}\left(\mu \lambda_{N}^{h}\right)$ such that:

$$
\left\{\begin{array}{c}
a\left(\tilde{u}^{h}, \tilde{v}^{h}\right)+b_{1}\left(\tilde{v}^{h}, \lambda_{N}^{h}\right)+b_{2}\left(\tilde{v}^{h}, \lambda_{T}^{h}\right)=\left(f^{h}, \tilde{v}^{h}\right)_{\tilde{V}}, \quad \forall \tilde{v}^{h} \in \tilde{V}^{h}, \\
\lambda_{N}^{h}=P_{M_{N}^{h}}\left(\lambda_{N}^{h}-r u_{n}^{h}\right), \\
\lambda_{T}^{h}=P_{M_{T}^{h}\left(\mu \lambda_{N}^{h}\right)}\left(\lambda_{T}^{h}-r u_{\tau}^{h}\right),
\end{array}\right.
$$

where $r>0$ and

$$
\begin{gathered}
M_{T}^{h}\left(\mu \lambda_{N}^{h}\right)=\left\{\delta^{h} \in X_{T}^{* h}, \quad\left\langle\delta^{h}, v^{h}\right\rangle_{\Gamma_{3}} \leq \int_{\Gamma_{3}} \mu \lambda_{N}^{h}\left|v_{\tau}^{h}\right| d \Gamma, v^{h} \in H_{\Gamma}\right\}, \\
M_{N}^{h}=\left\{\delta^{h} \in X_{n}^{* h}, \quad\left\langle\delta^{h}, v^{h}\right\rangle_{\Gamma_{3}} \leq 0, v^{h} \in K_{n}^{h}\right\},
\end{gathered}
$$

and where $P_{M}$ is the projection over $M$. For more details we refer to [27].

\subsection{Matrix formulation}

In this section, we adopt the same technical discretization as in the work [27]. Let $\mathbf{a}_{j}\left(j=1, \ldots, n_{c}\right)$ be a contact node (i.e. $\mathbf{a}_{j}$ are the nodes forming $\Gamma_{3}$ ). The displacement vector at $\mathbf{a}_{j}$ is denoted by $\mathbf{u}_{j}$, i.e. $\mathbf{u}_{j}=u\left(\mathbf{a}_{j}\right)$. Denoting $n_{j}$ and $\boldsymbol{t}_{j}$, the unit outward normal vector to $\Gamma_{3}$ and the unit tangential vector to $\Gamma_{3}$, respectively. Let us introduce the linear mappings

- $\mathbf{N}: \mathbb{R}^{2 d} \rightarrow \mathbb{R}^{n_{c}}$, such that $(\mathbf{N u})_{j}=\mathbf{u}_{j}^{\top} \boldsymbol{n}_{j}, j=1, \ldots, n_{c}$.

- $\mathbf{T}: \mathbb{R}^{2 d} \rightarrow \mathbb{R}^{n_{c}}$, such that $(\mathbf{T u})_{j}=\mathbf{u}_{j}-\left(\mathbf{u}_{j}^{\top} \boldsymbol{n}_{j}\right) \boldsymbol{n}_{j}=\left(\mathbb{I}_{d}-\boldsymbol{n}_{j} \boldsymbol{n}_{j}^{\top}\right) \mathbf{u}_{j}, j=1, \ldots, n_{c}$.

The finite element discretization leads to the following matrices and vectors: 
- $\mathbf{A},(2 d) \times(2 d)$ the elastic matrix (symmetric and positive definite) ;

- B, $d \times d$ electric potential stiffness matrix (symmetric positive definite);

- E, $d \times(2 d)$ coupling matrix;

- $\mathbf{M}_{n}$ and $\mathbf{M}_{\tau}$ normal and tangential mass matrices $\left(n_{c} \times n_{c}\right)$;

- $\mathbf{f}\left(\right.$ the external forces in $\left.\mathbb{R}^{2 d}\right), \mathbf{q}$ (the external charges in $\mathbb{R}^{d}$ ).

- $\lambda_{N}, \lambda_{T}$ the vectors associated to $\lambda_{N}^{h}$ and $\lambda_{T}^{h}$ respectively.

With the above notations, we can solve the Problem 8 with Coulomb friction using fixed point procedure on the friction threshold (see $[27,28]$ for more details). The Fixed point on the friction threshold procedure is in Uzawa Algorithm 2,

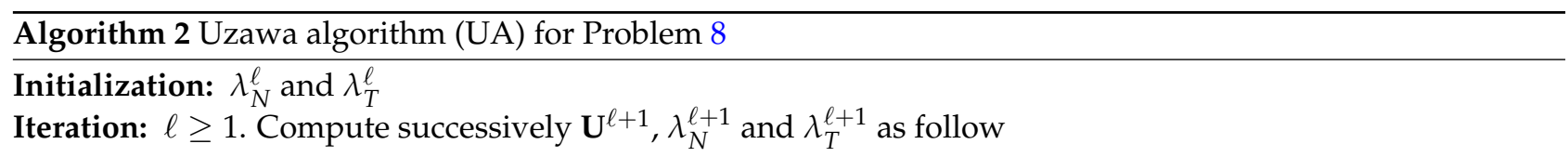

Step 1: $\mathbf{U}^{\ell+1}$ such that

Step 2: $\lambda_{N}^{\ell+1}$ such that

$$
\mathcal{A} \mathbf{U}^{\ell+1}=b^{\ell}
$$

Step 3: $\lambda_{T}^{\ell+1}$ such that

$$
\lambda_{N}^{\ell+1}=\left(r \mathbf{N u}^{\ell+1}-\lambda_{N}^{\ell}\right)^{+}
$$

$$
\lambda_{T}^{\ell+1}=P_{B\left(0,-\mathcal{F} \lambda_{N}^{\ell}\right)}\left(\lambda_{T}^{\ell}-r T \mathbf{u}^{\ell+1}\right)
$$

where

$$
\begin{gathered}
\mathbf{U}=\left[\begin{array}{l}
\mathbf{u} \\
\varphi
\end{array}\right], \mathcal{A}=\left[\begin{array}{cc}
\mathbf{A} & -\mathbf{E}^{\top} \\
\mathbf{E} & \mathbf{B}
\end{array}\right], \\
b^{\ell}=\left[\begin{array}{l}
\mathbf{b}_{1} \\
\mathbf{b}_{2}
\end{array}\right]=\left[\begin{array}{c}
\mathbf{f}+\mathbf{M}_{n} \mathbf{N} \lambda_{N}^{\ell}+\mathbf{M}_{\tau} \mathbf{T} \lambda_{T}^{\ell} \\
\mathbf{q}
\end{array}\right],
\end{gathered}
$$

$B\left(0,-\mathcal{F} \lambda_{N}^{\ell+1}\right)$ denote the ball of center 0 and radius $-\mathcal{F} \lambda_{N}^{\ell}>0$ and $x^{+}$denote the non negative part of $x$ i.e. $x^{+}=\max (0, x)$.

Remark 1. In practice, the Algorithm 2 is solved using Tresca friction with slip bound $S^{\ell}$ and an fixed point iteration is used to compute the problem with Coulomb friction, i.e. $S^{\ell+1}=-\mathcal{F} \lambda_{N}^{\ell}$.

To compute the solution of the system (70), we proceed by the following elimination technique:

$$
\begin{array}{r}
\varphi=\mathbf{B}^{-1} \mathbf{E} \mathbf{u}+\mathbf{B}^{-1} \mathbf{b}_{2}, \\
\mathbf{S}_{c} \mathbf{u}=\mathbf{b}_{1}-\mathbf{B}^{-1} \mathbf{b}_{2},
\end{array}
$$

where $\mathbf{S}_{c}$ is the Schur complement given by $\mathbf{S}_{c}=\mathbf{A}-\mathbf{E}^{\top} \mathbf{B}^{-1} \mathbf{E}$. Since the matrices $\mathbf{A}$ and $\mathbf{B}^{-1}$ are symmetric and positive definite, the suitable method for solving the subsystem (74) is the Conjugate Gradient method (CG). We take profit from the Schur complement $\mathbf{S}_{\mathcal{C}}$ to obtain a convenient preconditioner, as discussed in [16] and reference therein the (CG)-preconditioner is $\mathbf{P}=\mathbf{A}$. The preconditioned Conjugate Gradient method for solving the system (74) is stated in the Algorithm 3. Once $\mathbf{u}$ is computed one can compute $\varphi$ by the explicit formula (73).

The solution of the problem with Coulomb friction is stated in the algorithm 4

\subsection{Numerical example}

The algorithms are implemented in MATLAB on computer equipped running Windows 10 core i7 of 2.4GHz clock frequency and 6 GB RAM. As example, the domain consists of two-dimensional rectangular domain $\Omega=(0,2) \times(0,1)$ as in the Figure 1, with boundaries $\Gamma_{D}=\{0\} \times[0,1] \cup\{2\} \times[0,1], \Gamma_{3}=$ $[0,2] \times\{0\}$ and $\Gamma_{N}=[0,2] \times\{1\}$. External body force and charge are $f=0$ and $q=0$, respectively. On $\Gamma_{D}$ the 

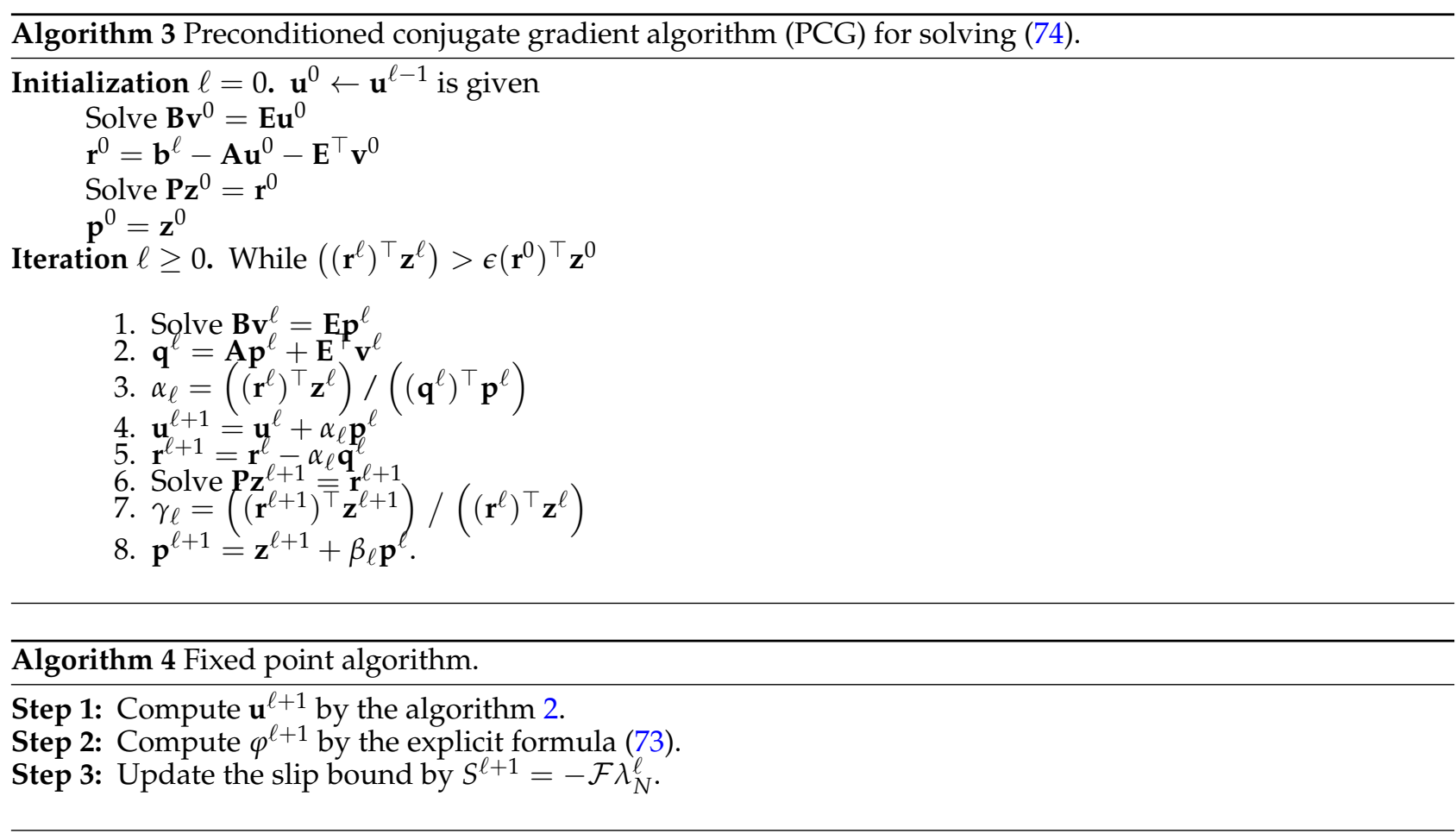

displacements and the electric potential are prescribed, i.e., $\mathbf{u}=0$ and $\varphi=0$ on $\Gamma_{D}$. On $\Gamma_{N}$, non-homogeneous Neumann boundary conditions are prescribed $f_{0}=\sigma(u) \cdot n=-2$. On $\{1\} \times(0,1)$ the homogeneous Neumann boundary condition is applied $(\sigma n=0$ and $D n=0)$. For seek of simplicity, the normalized gap between $\Gamma_{3}$ and the foundation is $g(\mathbf{x})=0$ and the friction coefficient is $\mathcal{F}=0.6$. The mesh is generated by using Matlab function "kmg.m" built by the author in [29].

The deformed configuration is showed in the Figure 2 and the the contour plot of the electric potential distribution are showed in the Figure 4 . The Figure 3 show the Lagrange multipliers $\lambda_{T, N}$ in the contact zone $\Gamma_{3}$ corresponding to the problem with Coulomb friction condition. The Figures 5 and 6 show the Lagrange multipliers $\lambda_{T, N}$ with different choices of loads acting on $\Gamma_{2}$, it is clear that the slide occurs when the load is important (large enough). The performance of the algorithm is presented in the Table 1 , as showed the number of iterations is independent of mesh refinement and the time of execution is significant.

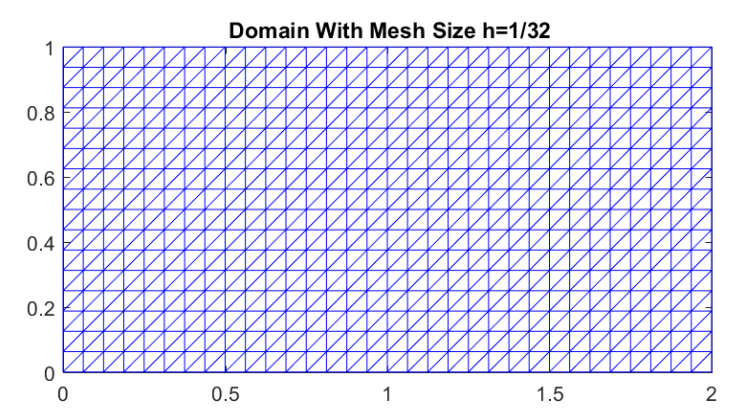

Figure 1. Initial configuration. 


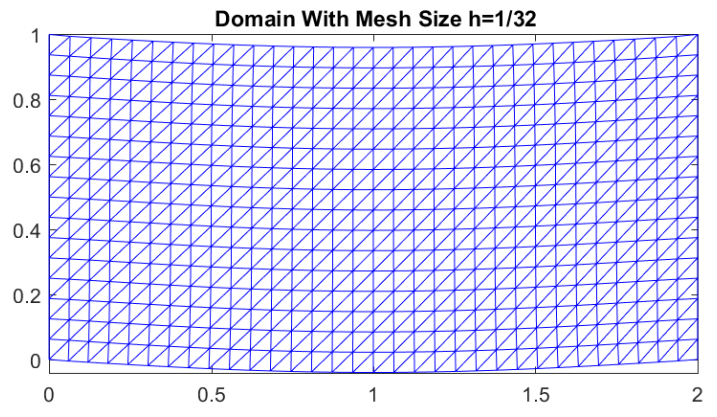

Figure 2. Deformed configuration.

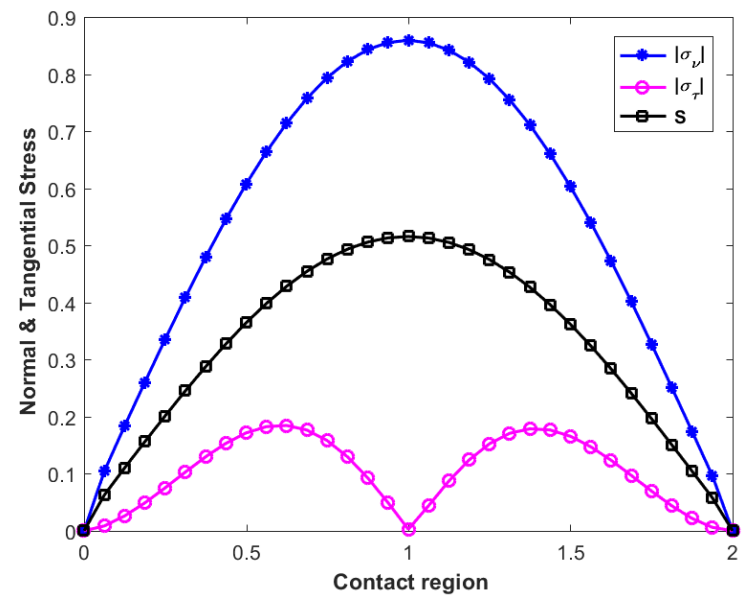

Figure 3. Multipliers for $\mathcal{F}=0.6$.

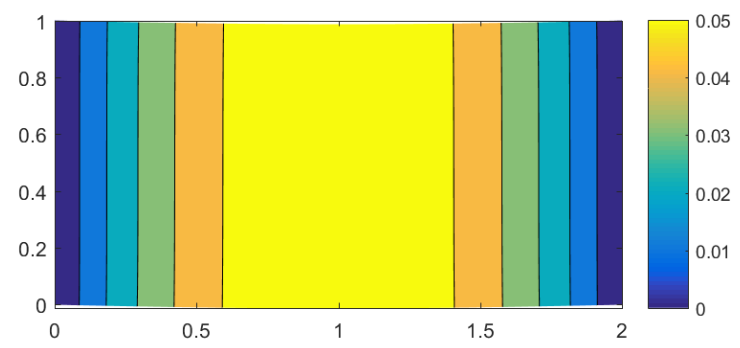

Figure 4. Contour plot of electic potential. 


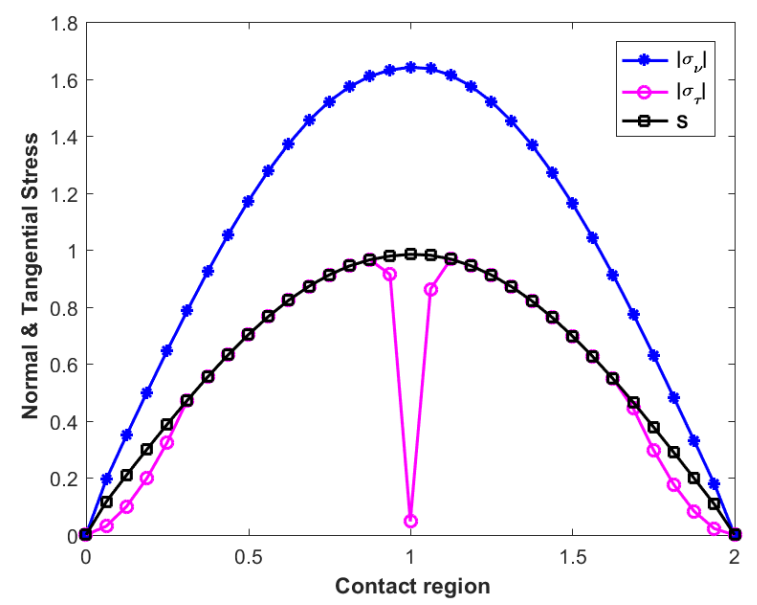

Figure 5. Multipliers with load -4 .

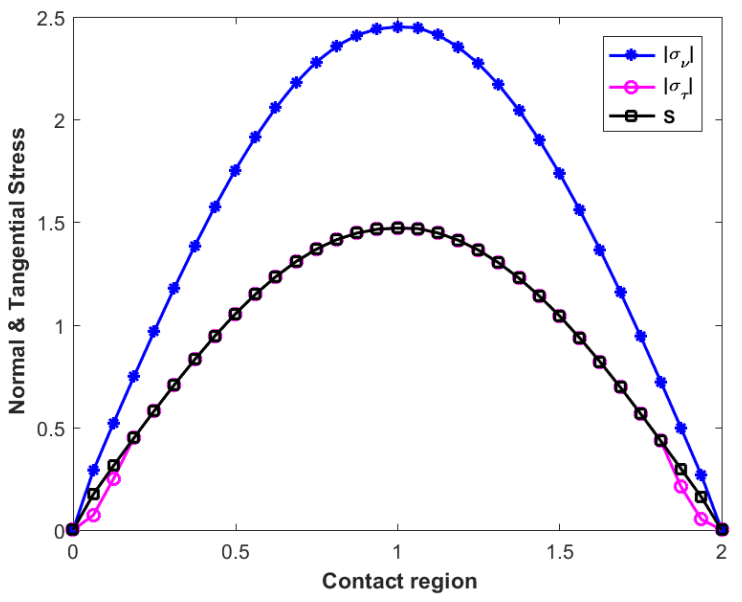

Figure 6. Multipliers with load -6 .

Table 1. Performance of the algorithms

\begin{tabular}{||ccccc||}
\hline Mesh size $h$ & $1 / 32$ & $1 / 64$ & $1 / 128$ & $1 / 256$ \\
\hline \hline Number of iterations of FP & 3 & 4 & 4 & 4 \\
Number of iterations of UA & 39 & 39 & 39 & 39 \\
Number of iterations of PCG & 2 & 2 & 2 & 2 \\
CPU time (seconds) & 0.1341 & 2.0863 & 21.1882 & 238.4585 \\
\hline
\end{tabular}




\section{Conclusion}

We have investigated numerical analysis of a model describing the process of contact between a piezoelectric body and non-conductive foundation. The behavior of the material is modeled with a electro-elastic constitutive law. The contact is formulated by Signorini conditions and Coulomb friction. In coming works, more general problem with non linear constitutive equation and non monotone friction will be treated. This is may be handled by fixed point iteration and hemivariational inequalities [30].

Author Contributions: All authors contributed equally to the writing of this paper. All authors read and approved the final manuscript.

Conflicts of Interest: "The authors declare no conflict of interest."

\section{References}

[1] Arnau, A., \& Soares, D. (2009). Fundamentals of piezoelectricity. In Piezoelectric transducers and applications (pp. 1-38). Springer, Berlin, Heidelberg.

[2] Katzir, S. (2006). The discovery of the piezoelectric effect. In the beginnings of piezoelectricity (pp. 15-64). Springer, Dordrecht.

[3] Bisegna, P., Maceri, F., \& Lebon, F. (2002). The unilateral frictional contact of a piezoelectric body with a rigid support. In Contact mechanics (pp. 347-354). Springer, Dordrecht.

[4] Taik, A., Essoufi, E., Benkhira, E., \& Fakhar, R. (2010). Analysis and numerical approximation of an electro-elastic frictional contact problem. Mathematical Modelling of Natural Phenomena, 5(7), 84-90.

[5] Shillor, M., Sofonea, M., \& Telega, J. J. (2004). Models and analysis of quasistatic contact: variational methods (Vol. 655). Springer Science \& Business Media.

[6] Sofonea, M., \& Essoufi, E. H. (2004). A piezoelectric contact problem with slip dependent coefficient of friction. Mathematical Modelling and Analysis, 9(3), 229-242.

[7] Sofonea, M., \& Matei, A. (2012). Mathematical models in contact mechanics (No. 398). Cambridge University Press.

[8] Barboteu, M., Fernández, J. R., \& Ouafik, Y. (2008). Numerical analysis of two frictionless elastic-piezoelectric contact problems. Journal of mathematical analysis and applications, 339(2), 905-917.

[9] Kikuchi, N., \& Oden, J. T. (1988). Contact problems in elasticity: a study of variational inequalities and finite element methods (Vol. 8). Siam.

[10] Ekeland, I., \& Temam, R. (1999). Convex analysis and variational problems (Vol. 28). Siam.

[11] Fortin, M., \& Glowinski, R. (2000). Augmented Lagrangian methods: applications to the numerical solution of boundary-value problems. Elsevier.

[12] Glowinski, R., \& Le Tallec, P. (1989). Augmented Lagrangian and operator-splitting methods in nonlinear mechanics (Vol. 9). SIAM.

[13] Glowinski, R., \& Marroco, A. (1975). Sur l'approximation, par éléments finis d'ordre un, et la résolution, par pénalisation-dualité d'une classe de problèmes de Dirichlet non linéaires. ESAIM: Mathematical Modelling and Numerical Analysis-Modélisation Mathématique et Analyse Numérique, 9(R2), 41-76.

[14] Stadler, G. (2004). Semismooth Newton and augmented Lagrangian methods for a simplified friction problem. SIAM Journal on Optimization, 15(1), 39-62.

[15] Essoufi, E. H., Fakhar, R., \& Koko, J. (2015). A decomposition method for a unilateral contact problem with Tresca friction arising in electro-elastostatics. Numerical Functional Analysis and Optimization, 36(12), 1533-1558.

[16] Essoufi, E. H., Koko, J., \& Zafrar, A. (2017). Alternating direction method of multiplier for a unilateral contact problem in electro-elastostatics. Computers \& Mathematics with Applications, 73(8), 1789-1802.

[17] Hüeber, S., Matei, A., \& Wohlmuth, B. I. (2005). A mixed variational formulation and an optimal a priori error estimate for a frictional contact problem in elasto-piezoelectricity. Bulletin mathématique de la Société des Sciences Mathématiques de Roumanie, 209-232.

[18] Matei, A. (2009). A Variational Approach for an Electro-Elastic Unilateral Contact Problem. Mathematical Modelling and Analysis, 14(3), 323-334.

[19] Matei, A., \& Sofonea, M. (2017). A mixed variational formulation for a piezoelectric frictional contact problem. IMA Journal of Applied Mathematics, 82(2), 334-354.

[20] Haslinger, J., \& Sassi, T. (2004). Mixed finite element approximation of 3D contact problems with given friction: error analysis and numerical realization. ESAIM: Mathematical Modelling and Numerical Analysis, 38(3), 563-578.

[21] Mandel, J. (1990). On block diagonal and Schur complement preconditioning. Numerische Mathematik, 58(1), 79-93.

[22] Brezzi, F., \& Fortin, M. (2012). Mixed and hybrid finite element methods (Vol. 15). Springer Science \& Business Media.

[23] Duvant, G., \& Lions, J. L. (2012). Inequalities in mechanics and physics (Vol. 219). Springer Science \& Business Media. 
[24] Rabel, R. G., Han, W., \& Sofonea, M. (2002). Quasistatic contact problems in viscoelasticity and viscoplasticity. American Mathematical Soc..

[25] Kinderlehrer, D., \& Stampacchia, G. (1980). An introduction to variational inequalities and their applications (Vol. 31). Siam.

[26] Voigt, W. (1928). Lehrbuch der kristallphysik (Vol. 962). Leipzig: Teubner.

[27] Khenous, H. B., Pommier, J., \& Renard, Y. (2004). Hybrid discretization of the Signorini problem with Coulomb friction. Theoretical aspects and comparison of some numerical solvers. A paraître Numerical Applied Mathematics.

[28] Laborde, P., \& Renard, Y. (2008). Fixed point strategies for elastostatic frictional contact problems. Mathematical methods in the applied sciences, 31(4), 415-441.

[29] Koko, J. (2015). A Matlab mesh generator for the two-dimensional finite element method. Applied Mathematics and Computation, 250, 650-664.

[30] Migórski, S., Ochal, A., \& Sofonea, M. (2012). Nonlinear inclusions and hemivariational inequalities: models and analysis of contact problems (Vol. 26). Springer Science \& Business Media.

(c) 2020 by the author; licensee PSRP, Lahore, Pakistan. This article is an open access article distributed under the terms and conditions of the Creative Commons Attribution (CC-BY) license (http://creativecommons.org/licenses/by/4.0/). 